\title{
Strategies for survival
}

\author{
The United Nation's Sustainable Development Goals contain a commitment to abolish world hunger. Sounds like
} a job for a plant scientist!

n September 2000, the United Nations hosted the largest gathering of world leaders at the Millennium Summit. Its avowed aim was to seize "a unique and symbolically compelling moment to articulate and affirm an animating vision for the United Nations". One thing that emerged was the adoption of the Millennium Development Goals (MDGs), within which all countries committed to substantially and measurably improve the lot of mankind by the year 2015 .

There was not actually a great deal relating to plant biology in those goals. True, goal 7 was to ensure environmental sustainability, but this concentrated mainly on reducing carbon dioxide emissions, forest loss and species extinctions.

However, goal 1 was to halve the proportion of undernourished people in the world, something that should surely require the skills of plant biologists. This was one of the more successful goals. By 2015, the undernourished constituted $12.9 \%$ of the global population, down from $23.3 \%$ in 1990 . Whether this was due to improvements in agriculture is, however, debateable as most of the gains were made in Asia and Latin America, concurrent with their rapid economic growth; China alone supplied almost two thirds of the total reduction.

Fast-forward to 2015, and with the conclusion of the MDGs came a further set of targets, this time enshrined in the 17 Sustainable Development Goals (SDGs), which are both more wide-ranging and more specific. The involvement of agriculture and plant biology has also become a little more prominent. SDG 2 is to "end hunger, achieve food security and improved nutrition, and promote sustainable agriculture". This is no new aspiration for plant biologists. While accepting the Nobel Peace prize in 1970, Norman Borlaug talked of the Green Revolution as winning "a temporary success in man's war against hunger and deprivation".

The SDGs have detailed targets for 2030: to double agricultural productivity and incomes of small-scale food producers; to ensure sustainable food production systems and implement resilient agricultural practices; and to maintain the genetic diversity of crops and their related wild species. Much can be achieved in these areas without changing the plants we use. Adjustments to farming practices can reduce the inefficiencies found at particular locations, ranging from improved irrigation, transport and storage infrastructures, to more extensive changes in approach such as no-till or organic systems. The system of rice intensification (SRI), for example, has been employed by over ten million farmers in Asia, Africa and the Middle East. It was developed in Madagascar in the 1980s by the French Jesuit, Father Henri de Laulanié and adapted for use in other countries during the 1990s and 2000s, in part by the work of Norman Uphoff of Cornell University, New York. The approach uses less water than a traditional paddy, keeping soil moist rather than inundated, and involves transplanting young seedlings into grids of greater spacing. In the North Indian province of Bihar, where many hundreds of thousands of hectares of rice are under SRI cultivation, the Bihari government reports $40 \%$ higher yields than for more traditional farming. But SRI is always adapted to local conditions, making direct comparisons difficult to perform.

Higher-tech solutions also exist for maximizing the amount of food produced in particular locations. For example, a number of companies in the United States, Europe, Taiwan and Singapore are addressing the problems of supplying food to urban areas by creating 'vertical farms'. This is indoor agriculture, with plants grown on trays or hanging planters, stacked up to take advantage of the full height as well as the breadth of space available. One of the leading vertical farming firms, AeroFarms, recently built the world's largest indoor vertical farm, its ninth, in Newark, New Jersey. Such units are extremely energy intensive, requiring artificial lighting, but this can often be supplied at least in part by solar panels and wind turbines outside the farms.

It is ironic that the same economic growth in Asia which helped the achievement of the MDGs in reducing undernourishment also created a greater demand for meat, especially beef. Raising cattle for food is considerably less efficient than growing crops for direct consumption. The feed conversion rate (FCR) of cattle is typically more than 6 , meaning that 6 times the amount of fodder has to be grown as beef produced. Other livestock species are less inefficient; pigs have an FCR of about 4, rabbits from $2.5-3$, and poultry around 2 . However, lower than all of these are crickets at 1.7. Insects are a common dietary component in many cultures throughout the world and insect protein is similar to that of shrimps and other crustaceans, so perhaps introducing more insects into the 'western' diet would help ease pressure on agricultural lands.

Ultimately, what Norman Borlaug described as the "Population Monster" will only be assuaged by increasing global crop production. Yet, our goals for a sustainable future will not be reached by using more of the planet's surface for agriculture. We must instead create higher-yielding crops, producing more usable product with lower inputs. Midterm solutions will involve breeding and engineering varieties that direct more of their gained resources into the parts of plants that we use.

Longer-term solutions will require crops whose efficiency is achieved through more radical means. We can look forward to seeing plants whose photosynthetic apparatus has been 'improved' by creating $\mathrm{C}_{4}$ varieties of $\mathrm{C}_{3}$ species, by adjusting the characteristics of their carbon-fixing enzyme RUBISCO, or by tweaking their mechanisms for non-photochemical quenching to better cope with high and fluctuating light. Other crop varieties with better nitrogen and phosphorous use will emerge through engineering of their symbioses with soil microbiota, thus reducing the need for industrially produced fertilizers - the manufacture of which is a major producer of greenhouse gasses. Water efficiency may be increased by engineering stomata, and salt tolerance by adjustment of ion transporters.

All the SDGs rely directly or indirectly on a freedom from want. None can be satisfied without the adequate supply of food and materials that plants produce. It is no exaggeration to say that if the plant sciences cannot provide a route to a sustainable future, nothing else will.

Published online: 5 December 2017 https://doi.org/10.1038/s41477-017-0081-x 